\title{
Designing Excited States: Theory-Guided Access to Efficient Photosensitizers for Photodynamic Action**
}

\author{
Yusuf Cakmak, Safacan Kolemen, Selin Duman, Yavuz Dede, Yusuf Dolen, Bilal Kilic, \\ Ziya Kostereli, Leyla Tatar Yildirim, A. Lale Dogan, Dicle Guc, and Engin U. Akkaya*
}

Photodynamic therapy (PDT) is a treatment modality for certain malignant (skin, head and neck, gastrointestinal, gynecological cancers), premalignant (actinic keratosis), and nonmalignant (psoriasis) indications. ${ }^{[1]}$ Broader acceptance by the medical community and applicability is hampered, at least in part, by the less than optimal photophysical characteristics of the porphyrin derivatives. This situation sparked a worldwide search for novel sensitizers leading to new compounds, some holding more promise than others. ${ }^{[2-4]}$ The primary cytotoxic agent involved in the photodynamic action is singlet oxygen $\left({ }^{1} \Delta_{\mathrm{g}}\right)$, the efficient generation of which is linked invariably to the intersystem crossing (ISC) efficiency of the excited sensitizer. Most organic dyes have low triplet quantum yields, and in many recent candidates for photodynamic sensitizers, heavy atoms are incorporated into the structure as a strategy to improve spin-orbit coupling leading to facilitated intersystem crossing. ${ }^{[5,6]}$ While this approach seems fail-safe, incorporation of heavy atoms such as bromine, iodine, selenium, and certain lanthanides very often leads to increased "dark toxicity" ${ }^{[7]}$ Unlike traditional chemotherapy agents, in principle, photodynamic therapy sensitizers themselves can be nontoxic, either at cellular or organ levels, even at relatively high concentrations. We have been interested in trying to find alternative ways of achieving increased intersystem crossing without the use of heavy atoms

[*] Y. Cakmak, S. Kolemen, B. Kilic, Prof. Dr. E. U. Akkaya UNAM-Institute of Materials Science and Nanotechnology Bilkent University, Ankara, 06800 (Turkey)

E-mail: eua@fen.bilkent.edu.tr

Z. Kostereli, Prof. Dr. E. U. Akkaya

Department of Chemistry

Bilkent University, Ankara, 06800 (Turkey)

S. Duman, Dr. Y. Dede

Department of Chemistry

Gazi University, Ankara, 06500 (Turkey)

Y. Dolen, Prof. Dr. A. L. Dogan, Prof. Dr. D. Guc

Department of Basic Oncology, Oncology Institute

Hacettepe University, Ankara, 06100 (Turkey)

Prof. Dr. L. T. Yildirim

Department of Engineering Physics

Hacettepe University, Ankara, 06800 (Turkey)

$[* *$ We are grateful for funding by the National Boron Research Institute (BOREN), the Turkish Academy of Sciences (TUBA), and State Planning Organization (DPT). Additional funding from TUBITAK (110T076 and 110T647) and GU-BAP is gratefully acknowledged. S.D. thanks TUBITAK for a scholarship.

D) Supporting information for this article is available on the WWWW under http://dx.doi.org/10.1002/anie.201105736. to minimize dark toxicity, turning our attention to the excitedstate properties of the sensitizers.

Designing efficient photoinduced ${ }^{1} \mathrm{O}_{2}$ generators requires that any existing operative fluorescence cycle of the fluorophore, which is through the $\mathrm{S}_{0} \rightarrow \mathrm{S}_{1} \rightarrow \mathrm{S}_{0}$ states, has to be perturbed so as to minimize or shut down the $S_{1} \rightarrow S_{0}$ deactivation, and switch to the triplet surface once $S_{1}$ is accessed. A general design principle for a favorable $S_{1} \rightarrow T_{1}$ transition from an electronic structure viewpoint would in principle require the structural and electronic compatibility of the $S_{1}$ and $T_{1}$ states to surpass that of the $S_{1}-S_{0}$ pair. Once multiple electronic states come into play, quantum mechanical calculations providing a detailed understanding of the electronic structure are extremely helpful. Multi-configurational self-consistent field (MCSCF) techniques are the stateof-the-art computational chemistry approaches, when near degeneracies and excited states are considered. These methods may not reach chemical accuracy $\left( \pm 2-3 \mathrm{kcal} \mathrm{mol}^{-1}\right)$ for computing total energies, but they are crucial for a qualitatively correct description of the excited states and are capable of providing a conceptually complete picture of the photophysics taking place. Therefore, we mainly employed a popular variant of MCSCF techniques; the complete active space SCF (CASSCF) method in combination with relatively large basis sets and different active spaces. Details of CASSCF calculations are provided in the Supporting Information.

Our calculations on the parent Bodipy (4,4-difluoro-4-bora-3a,4adiaza-s-indacene, Scheme 1) showed that natural orbital occupancies of the $\mathrm{S}_{1}$ state describe an open-shell singlet with essentially double $(>1.9)$ or zero $(<0.1)$ electrons for all orbitals except the highest occupied molecular orbital (HOMO) and the lowest unoccupied molecular orbital (LUMO) that are

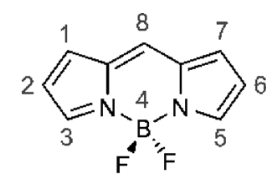

Scheme 1. Structure and numbering of the parent Bodipy compound. singly occupied (see the Supporting Information, Figure S1). It is no surprise to observe a fluorophore with low triplet quantum yield to have an excited state that possesses only two orbitals with single occupancy. Hence, to achieve our goal of efficient switching to the triplet manifold, we have to access excited states that differ from the ones that arise from simple HOMO $\rightarrow$ LUMO transitions. Among multiply excited configurations, doubly substituted ones are particularly important in enhancing $\mathrm{S}_{1}-\mathrm{T}_{1}$ coupling as shown by the seminal work of Salem and Rowland ${ }^{[8,9]}$ and the following work by Michl. ${ }^{[10]}$ Thus, the substitutions should invoke a simultaneous two-electron excitation from the 
ground state, guiding us to search for molecules with a pair of degenerate or near-degenerate occupied frontier orbitals that correlate with a similar virtual (unoccupied) pair and pointing out a design principle of orthogonal dimeric chromophores. This foundation has two important features: 1) Orthogonal placement prevents mixing of the $\pi$ systems of the two subunits leaving us with two essentially undisturbed chromophore cores; 2) Upon irradiation, both cores are almost equally likely to undergo a $\mathrm{HOMO} \rightarrow$ LUMO electron transfer (referring to the original monomer MOs), leading to an excited state mainly comprised of double substitutions.

Our previous involvement with Bodipy dyes led us to seek ways to implement these design considerations using these dyes. Bodipy dyes are exceptional fluorophores with amazing versatility ${ }^{[11-17]}$ and rich chemistry. ${ }^{[18-24]}$ Heavy-atom-functionalized Bodipy dyes showed some promise as potential sensitizers for photodynamic activity. ${ }^{[25-28]}$ It is also interesting to note that a few years ago, dimeric (albeit non-orthogonal) Bodipy derivatives with peculiar properties linked to exciton coupling between the chromophores were reported. ${ }^{[29]}$ We believe orthogonality to be an important distinction in our design. Our calculations on the orthogonal 8-2' dimer 3 clearly show (Figure S3) the essentially unperturbed Bodipy HOMO and LUMO as a result of lack of mixing of orthogonal $\pi$ frameworks. Orthogonality is secured by the strategic placement of the methyl substituents, resulting in a dihedral angle very close to $90^{\circ}$ for the two Bodipy cores. Orthogonal arrangement of the Bodipy units was also experimentally verified by the X-ray diffraction structure of the compound 3 (see below). The calculated energy differences $(2.96 \mathrm{eV}$, $2.97 \mathrm{eV}$ ) remain very close to the HOMO-LUMO gap of the Bodipy core $(3.10 \mathrm{eV})$, which further supports our claim of the Bodipy cores remaining essentially unperturbed in the dimeric form. Thus the orthogonal dimer should possess the desired features sought in the excitation process. CASSCF calculations on the orthogonal dimer $\mathbf{3}$ show that there is a huge amount of electronic reorganization upon $S_{0} \rightarrow S_{1}$ transition. $S_{1}$ with an equilibrium geometry very similar to $\mathrm{S}_{0}$ (Figure S3) lies $80 \mathrm{kcal} \mathrm{mol}^{-1}$ above the ground state (see the Supporting Information, Figure S5).

Computations of the $\mathrm{S}_{0} \leftrightarrow \mathrm{S}_{1}$ vertical transitions show that only $15 \mathrm{kcalmol}^{-1}$ of this difference is due to structural deformation, whereas electronic reorganization is more than four times as costly $\left(65 \mathrm{kcal} \mathrm{mol}^{-1}\right)$. This finding implies that the electronic structure of $S_{1}$ is rather unusual. Natural orbital occupation numbers show four odd electrons in four very similar orbitals (Figure S3). Thus, on excitation, both Bodipy units are essentially equally likely to undergo the inherent (Bodipy) HOMO $\rightarrow$ LUMO transition from HOMO- 1 to LUMO and HOMO to LUMO + 1 of Figure S3. In addition, orbitals optimized for the $S_{1}$ state support the involvement of both cores through their clearly visible delocalized character. Thus, the $S_{1}$ state could be predicted to be comprised of a linear combination of doubly substituted configurations (with respect to the closed-shell reference) and in line with this expectation, the CASSCF wave function is almost totally made up of doubly substituted configurations, an unexpectedly large value for any chromophore (see the Supporting Information, Figure S6, and the following discussion).
The dominance of the $S_{1}$ wave function by double substitutions is in stark contrast with the $\mathrm{S}_{0}$ wave function of the orthogonal dimer, as the latter is dominated by the reference configuration to an extent of approximately $90 \%$. These findings suggest a severe mismatch between the $S_{1}$ and $\mathrm{S}_{0}$ states and an enhanced transition to the $\mathrm{T}_{1}$ state lying $1.7 \mathrm{eV}$ lower (at $1.8 \mathrm{eV}$ with respect to the ground state). Essentially the same electronic structure fingerprints are observed for $8,8^{\prime}$ dimer 6 (see the Supporting Information, Figure S5). Repeated calculations with different active space basis set combinations without exception, showed a picture dominated by doubly substituted configurations and four singly occupied molecular orbitals in the $S_{1}$ state.

As a result, orthogonal functionalization of the Bodipy core by a second Bodipy is the key to switching to the triplet manifold. Based on these clearly encouraging computational results, we were highly motivated for the synthesis of a series of orthogonally linked dimeric Bodipys. The synthesis procedure for compound $\mathbf{3}$ is quite straightforward, especially in view of recent contributions to Bodipy chemistry by Jiao and co-workers. ${ }^{[30]}$ The parent compound $\mathbf{1}$ was formylated through a Vilsmeier reaction (Scheme 2). The Bodipy framework was then constructed around the formyl carbon atom

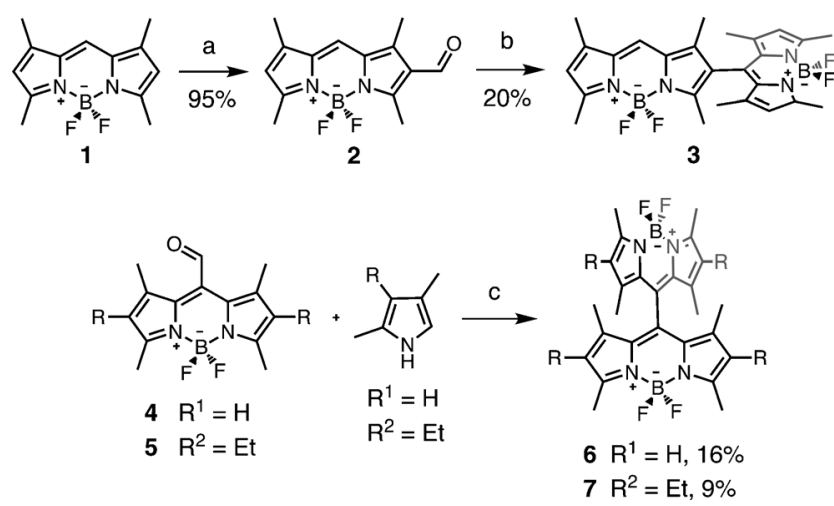

Scheme 2. Synthesis of the target photosensitizers. a) $\mathrm{POCl}_{3}, \mathrm{DMF}$, $\mathrm{ClCH}_{2} \mathrm{CH}_{2} \mathrm{Cl}, 50^{\circ} \mathrm{C}$; b) 2,4-dimethylpyrrole, trifluoroacetic acid (TFA), $\mathrm{CH}_{2} \mathrm{Cl}_{2}$, p-chloranil, $\mathrm{Et}_{3} \mathrm{~N}, \mathrm{BF}_{3} \cdot \mathrm{OEt}_{2}$; c) TFA, $\mathrm{CH}_{2} \mathrm{Cl}_{2}$, p-chloranil, $\mathrm{Et}_{3} \mathrm{~N}$, $\mathrm{BF}_{3} \cdot \mathrm{OEt}_{2}$.

using standard Bodipy chemistry. The absorption spectrum of the product showed a single band centered around $506 \mathrm{~nm}$, not much different than the parent compound. We were fortunate to be able to grow crystals appropriate for X-ray crystallographic analysis by slow evaporation of the dichloromethane solutions. The crystal structure obtained was consistent with our design expectations; the dihedral angle between the two Bodipy units was very close to $90^{\circ}$ (Figure 1), which was also predicted by the computational studies. Organic solutions of the orange-colored compound were noticeably lacking detectable fluorescence emission under ambient or hand-held UV lamp irradiation, an indication of competing excited state processes in action.

Recent progress in the derivatization of Bodipy dyes also allowed us to synthesize two different and more symmetrical $8,8^{\prime}$ orthogonal dimers. The recently reported ${ }^{[31]} 8$-formyl- 
a)

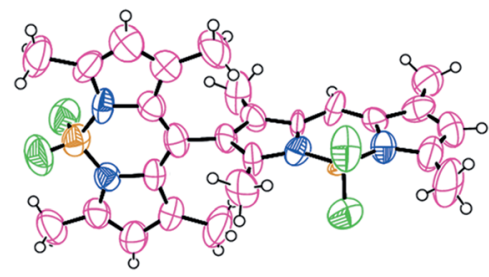

b)

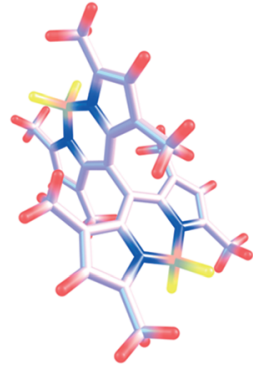

Figure 1. Structures of the dimeric Bodipys. a) ORTEP diagram (50\% probability level) for the X-ray diffraction structure of the orthogonal $8,2^{\prime}$ dimer 3. Dihedral angles between the two Bodipy units are almost $90^{\circ}\left(89.4^{\circ}\right)$; b) pptimized geometry of the orthogonal $8,8^{\prime}$ dimer 6 at the CAS(6e in 6o)/cc-pVDZ level. Dihedral angle between the two Bodipy units is almost $\left( \pm 0.5^{\circ}\right) 90^{\circ}$ in both dimers.

Bodipy derivative was transformed into the orthogonal dimers 6 and 7, using standard protocols (Scheme 2).

We surmised that a comparative study of compounds $\mathbf{1}, \mathbf{3}$, 6, and 7 would be instructive. First, we attempted to detect singlet-oxygen phosphorescence at $1270 \mathrm{~nm}$ in chloroform for all four compounds excited with a xenon-arc source at their respective absorption maxima; detection was carried out with a near-IR-sensitive detector. When excited at equal absorptivity concentrations for all compounds, dimer $\mathbf{3}$ gave the strongest singlet-oxygen phosphorescence emission (Figure 2) at the signature wavelengths (with a maximum around $1270 \mathrm{~nm}$ ); dimers 6 and $\mathbf{7}$ also showed phosphores-

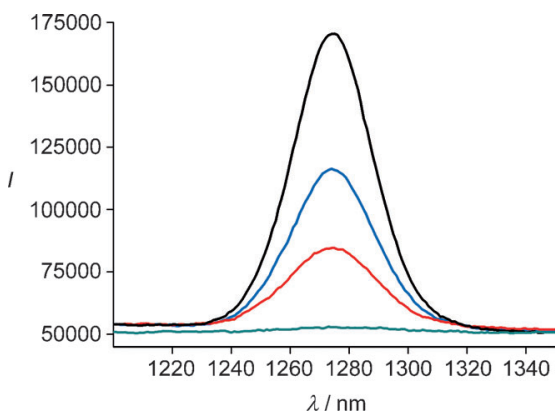

Figure 2. Singlet oxygen phosphorescence with sensitization from Bodipy derivatives: 1 (green), $\mathbf{3}$ (black), $\mathbf{6}$ (blue), and $\mathbf{7}$ (red) in $\mathrm{CHCl}_{3}$ at equal absorbances (0.2) at the wavelength of the maximum of their respective absorbances.

cence peaks, but with somewhat reduced intensity. On the other hand, no such emission was observed with compound $\mathbf{1}$ (Figure 2). That nonhalogenated Bodipy dyes have a low intersystem-crossing efficiency is a well-established fact: ${ }^{[7]}$ indeed that is at least part of the reason for their bright fluorescence and photostability. A list of relevant photophysical parameters for the sensitizers is given in Table 1 . It is interesting to note that for the dimers $\mathbf{6}$ and 7, significant fluorescence emission quantum yields are preserved, opening the possibility for a dual use as therapeutic and imaging agents. While the integral areas under the phosphorescence emission peak are a measure of singlet oxygen quantum yield,

Table 1: Comparative spectroscopic properties of Bodipy compounds.

\begin{tabular}{llllrl}
\hline Compound & $\lambda_{\text {abs }}{ }^{[\mathrm{a}]}[\mathrm{nm}]$ & $\lambda_{\mathrm{ems}}{ }^{[\mathrm{a}]}[\mathrm{nm}]$ & $\phi_{\mathrm{f}}$ & $\tau^{[\mathrm{ad}]}[\mathrm{ns}]$ & $\phi_{\Delta}{ }^{[\mathrm{e}]}$ \\
\hline $\mathbf{1}$ & 509 & 514 & $0.80^{[\mathrm{d}]}$ & 5.2 & - \\
$\mathbf{3}$ & 514 & 527 & $0.03^{[\mathrm{a}, \mathrm{b}]}$ & 2.5 & 0.51 \\
$\mathbf{6}$ & 515 & 588 & $0.37^{[\mathrm{a}, \mathrm{c}]}$ & 10.9 & 0.46 \\
$\mathbf{7}$ & 542 & 605 & $0.49^{[\mathrm{a}, \mathrm{c}]}$ & 5.0 & 0.21
\end{tabular}

[a] in $\mathrm{CHCl}_{3}$, [b] in reference to fluorescein in $0.1 \mathrm{M} \mathrm{NaOH}$ solution excited at $496 \mathrm{~nm}$, [c] in reference to rhodamine $6 \mathrm{C}$ in $\mathrm{EtOH}$ excited at $480 \mathrm{~nm},[\mathrm{~d}]$ in EtOH. [e] Singlet oxygen quantum yield was determined with respect to methylene blue (0.57 in dichloromethane). ${ }^{[32]}$

we opted for more quantitative assessment of singlet oxygen quantum yields using 1,3-diphenylisobenzofuran as a trap molecule and methylene blue as the reference compound (methylene blue has a singlet oxygen quantum yield of 0.57 under the conditions of the study in dichloromethane ${ }^{[32]}$ ). Excitation of the dyes was carried out by irradiation at the respective absorbance peak wavelengths with a monochromatized light source, at a fluence rate around $30 \mu \mathrm{W} \mathrm{cm}^{-2}$. The quantum yield for singlet oxygen generation for $\mathbf{3}$ is 0.51 in dichloromethane, which is much higher than for all nonhalogenated Bodipy dyes and many other organic chromophores and photosensitizers under comparable conditions. The other Bodipy dimers $\mathbf{6}$ and $\mathbf{7}$ also showed respectable singlet oxygen quantum yields of 0.46 and 0.21 , respectively.

For further demonstration of the singlet generation capacity and photocytotoxicity of the most active dimeric dye (compound $\mathbf{3}$ ), we carried out a cell culture assay with cancer cell lines. To that end, we prepared a micellar formulation of the 8,2'-orthogonal dimeric dye using Cremophor-EL (see the Experimental Section). ${ }^{[2]}$ The size distribution of the micelles was determined using electrophoretic light scattering (see the Supporting Information, Figure S7). The size distribution reveals a median size of $100 \mathrm{~nm}$ for the micellar constructs. Micelle-embedded dyes retained high levels of singlet oxygen generation capacity as revealed by another singlet oxygen trapping experiment, this time in aqueous media, using a water-soluble anthracene derivative (see the Supporting Information, Figure S13). Experimental verification of photocytotoxicity was carried out as follows: varying concentrations of the dimeric dye in Cremophor-EL micelles were incubated with K562 human erythroleukemia cells within a standard culture medium at $37^{\circ} \mathrm{C}$ in a humidified incubator containing $5 \% \mathrm{CO}_{2}$. Cells were irradiated with a green light-emitting diode (LED) source for $4 \mathrm{~h}$, followed by $44 \mathrm{~h}$ of incubation in the dark. The control group was incubated in the dark under otherwise identical conditions. The cell viabilities were determined using a standard MTT assay (Figure 3). Even at very low concentrations of dyes (as embedded within micelles), a significant decrease of the cell viability was observed (green bars in Figure 3) with a remarkable $\mathrm{EC}_{50}$ of $50 \mathrm{~nm}$. No statistically significant change was observed when the cells were kept in the dark, in the presence of the same concentration of the photosensitizer $\mathbf{3}$ (black bars in Figure 3). Photocytotoxicity was also revealed (Figure 4) using confocal microscopy with two fluorescent probes for cell viability (acridine orange and propidium iodide). Cells incubated with the sensitizer $\mathbf{3}$ in the dark show 


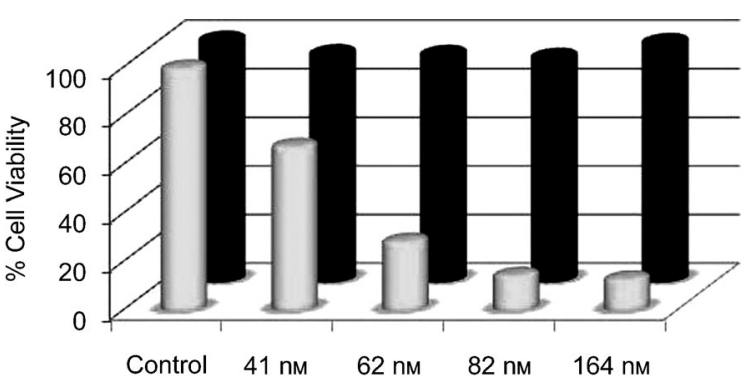

Figure 3. Photocytotoxicity of the sensitizer $\mathbf{3}$ as demonstrated by MTT assay. Cell suspensions (K562, human erythroleukemia cells) were seeded in 96-well flat-bottom plates and varying concentrations of the sensitizers were added to each well. Cells were kept either in the dark (back row), or under illumination with a green $(520 \mathrm{~nm})$ LED array at $2.5 \mathrm{~mW} \mathrm{~cm}^{-2}$ flow rate (front row) for a period of $4 \mathrm{~h}$ at $37^{\circ} \mathrm{C}$ in a humidified incubator containing $5 \% \mathrm{CO}_{2}$.

no changes as revealed by differential staining with the neutral and cell-permeable acridine orange. These cells appear bright green when excited at $488 \mathrm{~nm}$. Cationic propidium iodide stains these cells only when the membrane integrity is compromised; this happens only when both green LED irradiation and the sensitizer are acting on the cells. The use of excess propidium iodide results in red fluorescence in dead cells, or in cells dying through either apoptotic or necrotic pathways.

Thus, our work for the first time demonstrated a practical example of excited state design leading to efficient cytotoxic singlet oxygen generation with a potential in photodynamic therapy. With a sound theoretical framework established in their design, it is only natural to expect other photosensitizers to emerge with a similar line of reasoning. In this particular proof-of-principle work, excitation outside the therapeutic window is needed, but that issue can be easily addressed by the use of upconverting or persistent luminescence nanoparticles as secondary excitation sources. The other alternative is extending the conjugation in the dyes and exploring the longer wavelength extremes of the design principle employed herein. Our work along these lines is in progress.

\section{Experimental Section}

Cremophor-EL solubilization: Photosensitizer $3\left(5 \times 10^{-5} \mathrm{~mol}\right)$ was dissolved in THF $(1 \mathrm{~mL})$, treated with Cremophor EL (CrEL, $150 \mathrm{mg}$ ), and sonicated for $30 \mathrm{~min}$. THF was removed under reduced pressure, the remaining oil was dissolved in phosphate-buffered saline (PBS; $5 \mathrm{~mL}$ ), filtered through a $0.2 \mu \mathrm{m}$ membrane filter, and filled up to $10 \mathrm{~mL}$ with $\mathrm{PBS}(1 \times, \mathrm{pH} 7.4)$.

Cell culture and MTT assay: K562 human erythroleukemia cells (ATCC) were cultured in $25 \mathrm{~cm}^{3}$ culture flasks containing RPMI 1640 medium supplemented with heat-inactivated $10 \%$ fetal bovine serum, $2 \mathrm{~mm}$ L-glutamine, 100 units $\mathrm{mL}^{-1}$ penicillin $\mathrm{G}$, and $100 \mu \mathrm{gL}^{-1}$ streptomycin at $370{ }^{\circ} \mathrm{C}$ in a humidified incubator containing $5 \%$ $\mathrm{CO}_{2}$. Micellar preparation of the sensitizer was dissolved in RPMI 1640 medium and test concentrations were prepared daily. The 3-(4,5dimethylthiazol-2-yl)-2,5-diphenyltetrazolium bromide (MTT) assay was used to evaluate cell viability. Briefly, $50 \mu \mathrm{L}$ cell suspensions in RPMI 1640 medium containing $4 \times 10^{4} \mathrm{~K} 562$ cells were seeded in 96well flat-bottom plates (Costar, Cambridge, MA), and $50 \mu \mathrm{L}$ of varying concentrations of Cremophor-EL solubilized dye 3 in RPMI
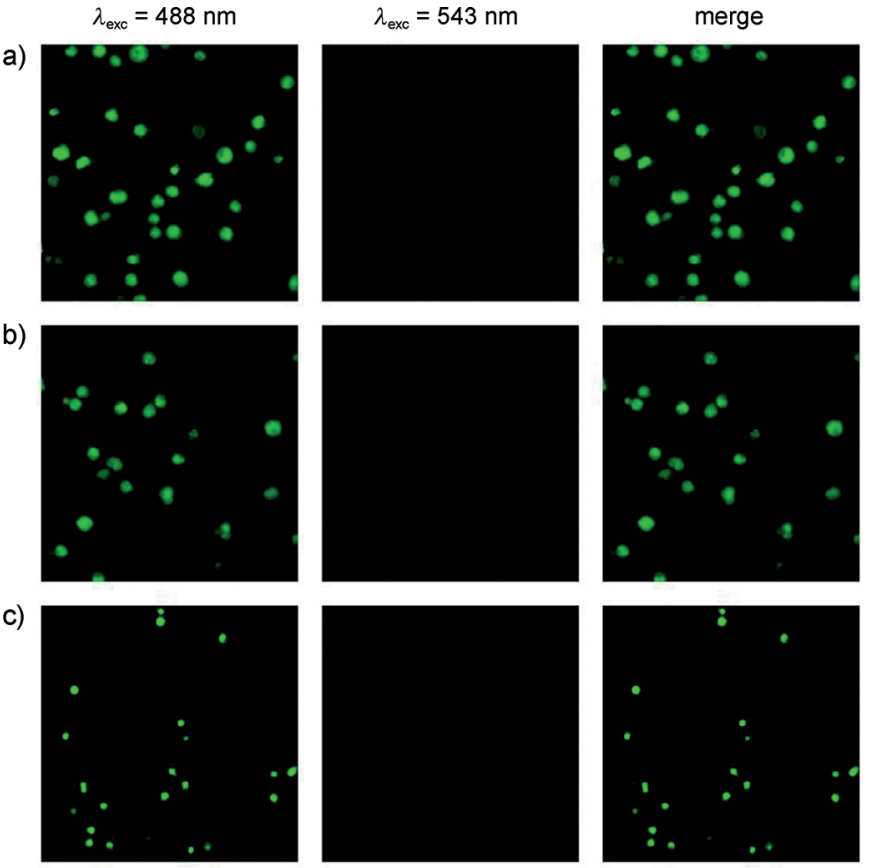

d)
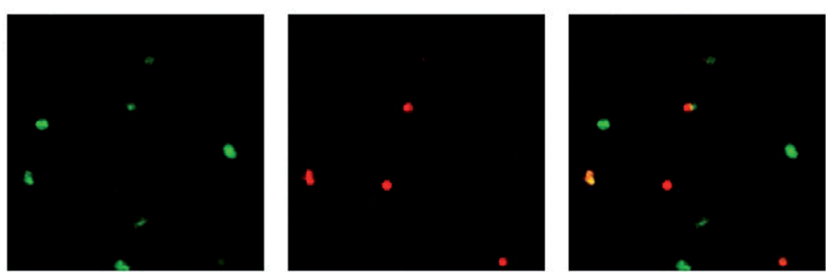

Figure 4. Photocytotoxic activity of the dimeric Bodipy 3 visualized by confocal microscopy. Cell suspensions (K562, human erythroleukemia cells) were seeded in 24-well plates. a) Cells in control-1-wells (upper plates) were incubated in the dark for $24 \mathrm{~h}$; b) cells in control-2-wells (second row plates) were incubated with $500 \mu \mathrm{L} /$ well Cremophor-EL solubilized sensitizer 3 (at a final concentration of $164 \mathrm{~nm}$ ) and were kept in the dark for $24 \mathrm{~h}$ in the same incubator; c) cells in control-3wells were illuminated for $4 \mathrm{~h}$ without the sensitizer and incubated for a further $20 \mathrm{~h}$ in the dark in the incubator; d) cells in control-4-wells (bottom row of plates) were illuminated for $4 \mathrm{~h}$ after addition of $164 \mathrm{~nm}$ dye 3 and were incubated for a further $20 \mathrm{~h}$ in the dark in the incubator. Cells in the wells were collected after $24 \mathrm{~h}$ and were imaged at $40 \times$ magnification. Live cells are preferentially stained with acridine orange (AO, green) (a), whereas dead cells are preferentially stained with propidium iodide ( $\mathrm{PI}$, red) because of increased cellular permeability (b).

1640 medium (final concentrations: $41 \mathrm{~nm}-164 \mathrm{~nm}$ ) were added into each well. All dye concentrations were tested three times in triplicate. Then, cells were kept either in the dark or were illuminated with a green $(520 \mathrm{~nm}) \mathrm{LED}$ array at $2.5 \mathrm{~mW} \mathrm{~cm}^{-2}$ fluence rate for a period of $4 \mathrm{~h}$ at $370^{\circ} \mathrm{C}$ in a humidified incubator containing $5 \% \mathrm{CO}_{2}$. To evaluate the cytotoxicity of the dye, plates that had been irradiated for $4 \mathrm{~h}$ were kept in the dark for a further $44 \mathrm{~h}$ and then MTT solution was added for measuring cell viability. As control, plates kept in the dark for $4 \mathrm{~h}$ were also incubated for an additional $44 \mathrm{~h}$. After $48 \mathrm{~h}$, $25 \mu \mathrm{L}$ of MTT solution (1.0 $\mathrm{mg} \mathrm{mL}^{-1}$ final concentration; Sigma Chemical Co., St. Louis, MO) were added to each well, and the plates were incubated for further $4 \mathrm{~h}$. The formazan precipitate was solubilized by adding lysing buffer $(80 \mu \mathrm{L}, \mathrm{pH} 4.7)$ composed of $23 \%$ SDS (sodium dodecyl sulfate) dissolved in a solution of $45 \%$ $N, N$ - dimethylformamide (DMF). After incubation overnight at $370^{\circ} \mathrm{C}$, the absorbances were read at $570 \mathrm{~nm}$ using a microplate 
reader (Spectramax Plus, Molecular Devices, Sunnyvale, California, USA). Cells incubated in culture medium alone served as control for cell viability (nontreated wells) either on the irradiated plate or on a plate that was kept in the dark. Cell viability (\%) was calculated as optical density (OD) of treated wells/OD of nontreated cells $\times 100$.

Preparation of stock solutions for fluorescence microscopy: A stock solution of propidium iodide (PI; Sigma P-4170) was prepared in distilled water, and used at a concentration of $0.5 \mathrm{mg} \mathrm{mL}^{-1}$. Acridine orange $(\mathrm{AO})$ was dissolved in phosphate buffered saline at a concentration of $100 \mu \mathrm{gmL}^{-1}$. One tablet of phosphate buffered saline (PBS; Amresco) was dissolved in distilled water to give a pH 7.4 buffered solution.

Confocal microscopy: Confocal microscopic analysis was also performed to evaluate cell viability. Briefly, $1500 \mu \mathrm{L}$ cell suspensions in RPMI 1640 medium containing $2 \times 10^{5} \mathrm{~K} 562$ cells/well were seeded in 24-well plates. Cells in the control-1-well were incubated in the dark for $24 \mathrm{~h}$ at $370^{\circ} \mathrm{C}$ in a humidified incubator containing $5 \% \mathrm{CO}_{2}$. Cells in the control-2-well were incubated with $500 \mu \mathrm{L} /$ well Cremophor-EL solubilized dye 3 (final dye concentration: $164 \mathrm{~nm}$ ) and were kept in the dark for $24 \mathrm{~h}$ in the same incubator. Cells in the control-3well were illuminated for $4 \mathrm{~h}$ without dye and incubated for a further $20 \mathrm{~h}$ in the dark in the incubator. Cells in control-4-well were illuminated for $4 \mathrm{~h}$ after addition of $164 \mathrm{~nm}$ dye 3 and were incubated for a further $20 \mathrm{~h}$ in the dark in the incubator. All conditions were studied in duplicate. Cells in wells were collected after $24 \mathrm{~h}$ and confocal microscopy images were acquired.

Received: August 13, 2011

Published online: October 20, 2011

Keywords: Bodipy dyes · fluorescence - photodynamic therapy sensitizers $\cdot$ singlet oxygen

[1] S. B. Brown, E. A. Brown, I. Walker, Lancet Oncol. 2004, 5, $497-$ 508.

[2] J. F. Lovell, T. W. B. Liu, J. Chen, G. Zheng, Chem. Rev. 2010, 110, 2839-2857.

[3] R. Bonnett, Chem. Soc. Rev. 1995, 24, 19-33.

[4] A. P. Castano, P. Mroz, M. R. Hamblin, Nat. Rev. Cancer 2006, 6, $535-545$.

[5] N. Adarsh, R. R. Avirah, D. Ramaiah, Org. Lett. 2010, 12, 5720 5723.

[6] T. Yogo, Y. Urano, Y. Ishitsuka, F. Maniwa, T. Nagano, J. Am. Chem. Soc. 2005, 127, $12162-12163$.

[7] S. H. Lim, C. Thivierge, P. Nowak-Sliwinska, J. Han, H. van den Bergh, G. Wagnieres, K. Burgess, H. B. Lee, J. Med. Chem. 2010 , $53,2865-2874$.
[8] L. Salem, C. Rowland, Angew. Chem. 1972, 84, 86-106; Angew. Chem. Int. Ed. Engl. 1972, 11, 92-111.

[9] L. Salem in Electrons in chemical reactions: First principles, Wiley, New York, 1982

[10] J. Michl, J. Am. Chem. Soc. 1996, 118, 3568-3579.

[11] R. Ziessel, G. Ulrich, A. Harriman, New J. Chem. 2007, 31, 496501.

[12] R. Ziessel, A. Harriman, Chem. Commun. 2011, 47, 611-631.

[13] O. A. Bozdemir, R. Guliyev, O. Buyukcakir, S. Selcuk, S. Kolemen, G. Gulseren, T. Nalbantoglu, H. Boyaci, E. U. Akkaya, J. Am. Chem. Soc. 2010, 132, 8029-8036.

[14] S. Kolemen, O. A. Bozdemir, Y. Cakmak, G. Barin, S. Ertan-Ela, M. Marszalek, J. H. Yum, S. M. Zakeeruddin, M. K. Nazeeruddin, M. Grätzel, E. U. Akkaya, Chem. Sci. 2011, 2, 949-954.

[15] O. A. Bozdemir, Y. Cakmak, F. Sozmen, T. Ozdemir, A. Siemiarczuk, E. U. Akkaya, Chem. Eur. J. 2010, 16, 6346-6351.

[16] I. Yildiz, S. Impellizzeri, E. Deniz, B. McCaughan, J. F. Callan, F. M. Raymo, J. Am. Chem. Soc. 2011, 133, 871-879.

[17] H. J. Kim, S. H. Kim, J. H. Kim, E-H. Lee, K-W. Kim, J. S. Kim, Bull. Korean Chem. Soc. 2008, 29, 1831-1834.

[18] A. Loudet, K. Burgess, Chem. Rev. 2007, 107, 4891-4932.

[19] G. Ulrich, R. Ziessel, A. Harriman, Angew. Chem. 2008, 120, 1202-1219; Angew. Chem. Int. Ed. 2008, 47, 1184-1201.

[20] Y. Cakmak, E. U. Akkaya, Org. Lett. 2009, 11, 85-88.

[21] O. Buyukcakir, O. A. Bozdemir, S. Kolemen, S. Erbas, E. U. Akkaya, Org. Lett. 2009, 11, 4644-4647.

[22] G. Ulrich, C. Goze, M. Guardigli, A. Roda, R. Ziessel, Angew. Chem. 2005, 117, 3760-3764; Angew. Chem. Int. Ed. 2005, 44, $3694-3698$.

[23] X. Qi, S. K. Kim, E. J. Jun, L. Xu, S.-J. Kim, J. Yoon, Bull. Korean Chem. Soc. 2007, 28, 2231-2234

[24] J. Killoran, L. Allen, J. F. Gallapher, W. M. Gallapher, D. F. O'Shea, Chem. Commun. 2002, $1862-1863$.

[25] S. O. McDonnell, M. J. Hall, L. T. Allen, A. Byrne, W. M. Gallagher, D. F. O'Shea, J. Am. Chem. Soc. 2005, 127, 1636016361.

[26] S. Atilgan, Z. Ekmekci, A. L. Dogan, D. Guc, E. U. Akkaya, Chem. Commun. 2006, 4398-4400.

[27] S. Ozlem, E. U. Akkaya, J. Am. Chem. Soc. 2009, 131, 48-49.

[28] S. Erbas, A. Gorgulu, M. Kocakusakogullari, E. U. Akkaya, Chem. Commun. 2009, 4956-4958.

[29] B. Ventura, G. Marconi, M. Broring, R. Kruger, L. Flamigni, New J. Chem. 2009, 33, 428-438.

[30] L. Jiao, C. Yu, J. Li, Z. Wang, M. Wu, E. Hao, J. Org. Chem. 2009, 74, $7525-7528$

[31] K. Krumova, G. Cosa, J. Am. Chem. Soc. 2010, 132, 1756017569 .

[32] M. Mirenda, C. A. Strassert, L. E. Dicelio, E. San Roman, ACS Appl. Mater. Interfaces 2010, 2, 1556-1560. 